\title{
Diabetic Neuropathy Collection: Progress in Diagnosis and Screening
}

\author{
Nikolaos Papanas (1)
}

Received: January 27, 2020 / Published online: February 28, 2020

(c) The Author(s) 2020

Keywords: Diabetes; Diabetic neuropathy; Diagnostic testing; Pain; Type 1 diabetes; Type 2 diabetes

VG: Victoria Glasson (Managing Editor of Diabetes Therapy).

NP: Nikolaos Papanas (Advisory Board Member of Diabetes Therapy).

This podcast is intended for medical professionals only.

VG: Hello and welcome to the Diabetes Therapy podcast. My name is Victoria Glasson and I am the Editor for Diabetes Therapy. Today we are talking to Professor Nikolaos Papanas who is a member of our Advisory Board. We are continuing our Diabetic Neuropathy podcast

Enhanced Digital Features To view enhanced digital features for this article go to https://doi.org/10.6084/ m9.figshare.11688375.

Electronic Supplementary Material The online version of this article (https://doi.org/10.1007/s13300020-00776-3) contains supplementary material, which is available to authorized users.

N. Papanas $(\bowtie)$

Second Department of Internal Medicine, Diabetes Centre-Diabetic Foot Clinic, Democritus University of Thrace, Alexandroupolis, Greece e-mail: papanasnikos@yahoo.gr collection, discussing diagnosis and screening of diabetic peripheral neuropathy.

NP: My name is Nikolaos Papanas. I am Professor of Internal Medicine-Diabetes Mellitus, Head of Diabetes Centre-Diabetic Foot Clinic, 2nd Department of Internal Medicine, Democritus University of Thrace, Alexandroupolis, Greece and Vice-President of the European Diabetic Foot Study Group.

VG: Why is diabetic peripheral neuropathy important?

NP: Diabetic peripheral neuropathy (DPN) is the commonest form of diabetic neuropathy and the most frequent manifestation of diabetes mellitus in the nervous system. It is chronic with a "stocking and glove distribution". It represents a pivotal aetiological factor of diabetic foot ulcerations through three mechanisms: (a) sensory impairment or loss, leading to tissue damage through unperceived traumas; (b) impaired biomechanics with plantar pressure redistribution and abnormalities in standing and walking through muscular atrophy; (c) dry, fragile skin with reduced sweating through impaired sweat gland innervation (also called sudomotor dysfunction). Moreover, DPN may lead to chronic severe neuropathic pain with a negative impact on quality of life.

VG: Why is early diagnosis of DPN important?

NP: DPN needs to be diagnosed early for several reasons. First, patients with DPN should be offered intensified education on foot hygiene 
(e.g. never walking barefoot, daily foot inspection) and, ideally, podiatric care and proper footwear. These measures aim to reduce foot ulcerations as complications of DPN. Secondly, they should be instructed on avoiding other neurotoxic factors, such as excessive alcohol. Moreover, improved glycaemic control without hypoglycaemias and glucose fluctuations, management of blood pressure and dyslipidaemia, smoking cessation-all these measures, which are, indeed, necessary for every subject with diabetes-should be offered more zealously. All these therapeutic priorities render timely diagnosis of DPN very important.

VG: How is DPN diagnosed?

NP: Scientific associations of diabetes recommend that DPN is diagnosed based on careful comprehensive bedside clinical examination. The latter should assess both sensory (which are, generally, affected earlier) and motor modalities (which are thought to be affected at later stages). Moreover, it should assess both small (pain and temperature perception) and large nerve fibres (remaining functions). To do this, simple tools are required: a patella hammer, a graded $128-\mathrm{Hz}$ tuning fork, a sterile test strip, a rod for hot/cold discrimination, and, secondarily, a 10-g monofilament and some cotton wool. After some training, the everyday clinician can easily carry out this examination. To promote comprehensiveness and to facilitate comparison of clinical findings, there are some clinical examination scores: among these, the most widely used is the neuropathy disability score (NDS).

Scientific associations of diabetes also recommend that nerve conduction study is not routinely used but reserved for special circumstances, e.g. research purposes, atypical clinical manifestation, or differential diagnosis from other causes of neuropathy.

VG: Why do we need new diagnostic tests?

NP: New tests aim to improve the early diagnosis of DPN. This can be accomplished in two ways: either the new tests detect signs of DPN before its clinical diagnosis or they aim to simplify the procedure, so that more subjects with diabetes can be screened, even by trained nurses. Ideally, a test should do both. Some of the new tests are highly sophisticated and demand specialised techniques and personnel, while others are very simple and can be easily and quickly carried out.

VG: What are the new diagnostic tests for DPN?

NP: New diagnostic tests may be classified into those evaluating small nerve fibres and those evaluating large nerve fibres. Tests of small nerve fibres include the indicator test for sudomotor function Neuropad, corneal confocal microscopy of the human eye, skin biopsy for assessment of intra-epidermal nerve fibres, Sudoscan, LDIflare, and the non-commercially available Neuroquick. Tests of large nerve fibres include automated sural nerve conduction study with a special portable device, Vibratip for vibration perception, Ipswich Touch Test, and the non-commercially available steel ballbearing.

\section{(a) Tests For Small Fibre Function}

The indicator test Neuropad is a plaster assessing sweat gland function (sudomotor function). This is accomplished by evaluating the sweat produced by the feet. Indeed, sweat contains water, which reacts with the blue salt cobalt dichloride* of the plaster and changes its colour to pink. The test is very simple: all you have to do is attach it to the plantar surface of the feet to a callus-free area between the 1st and 2nd metatarsal heads bilaterally and then wait for $10 \mathrm{~min}$ to read the colour change: complete bilateral colour change from blue to pink within $10 \mathrm{~min}$ is the normal response, while absent or incomplete colour change is abnormal. The procedure is so simple that it can be carried out by any individual, including patients themselves for self-examination. Moreover, we have shown excellent reproducibility. We have also found that the test, by virtue of its visual nature, increases motivation of subjects with diabetes to be instructed on foot care.

Neuropad has been demonstrated to yield very high sensitivity and negative predictive value with modestly high specificity and positive predictive value. Therefore, it has been proposed as a screening tool primarily to exclude DPN. Its absolute simplicity adds to its value. We have also reported its potential utility 
in diagnosing DPN 5 years earlier than clinical examination in type 2 diabetes.

Interestingly, Neuropad has been used for research purposes as well. Measurement of the absolute time until colour change has been shown to correlate with clinical and electrophysiological severity of DPN. Finally, novel automated image analysis software has been shown to improve the diagnostic performance of Neuropad.

Corneal confocal microscopy (CCM) quantitatively evaluates small nerve fibres in the cornea of the human eye. It can study several nerve fibre parameters and requires expensive equipment and specialised personnel. Thus, it is currently used by expert groups mainly for research purposes. Generally, its sensitivity and specificity are moderate to high. It can detect nerve fibre abnormalities early in the course of diabetes. Importantly, CCM has been shown to detect improvements in DPN after therapeutic interventions, notably simultaneous pancreas and kidney transplantation or intensified glycaemic control. Therefore, it is hoped that it will improve our insights into the natural history of DPN. It is non-invasive and has high reproducibility.

Skin biopsy is minimally invasive. It quantitatively evaluates small nerve fibres in the skin of the lower extremities. Like CCM, it can study several nerve fibre parameters, it can detect nerve fibre abnormalities early in the course of diabetes, and it is used by expert groups mainly for research purposes. Generally, its sensitivity and specificity are moderate to high. A worldwide reference study has reported useful normative data for skin biopsy parameters.

Sudoscan again assesses sudomotor function without requiring specialised personnel. It is a device measuring the low-voltage electrical current, which attracts sodium chloride from sweat produced by palms in the hands and soles in the feet. It is non-invasive and reproducible. It has high sensitivity and moderate to high specificity.

Neuroquick is a portable device with a fan. The latter emits cold air with adjustable fan velocity. It assesses cold air sensation on the foot dorsum. It has been suggested as a very accurate screening tool for early detection of small fibre impairment.

LDIflare employs a modified laser Doppler imager to measure the flare size in foot dorsal skin after heating. It is simple and non-invasive. High sensitivity and specificity for DPN, as well as age-dependent normative values have been reported.

\section{(b) Tests For Large Fibre Function}

Automated nerve conduction study is carried out with the portable device $\mathrm{NC}$-stat ${ }^{\circledR}$ DPNCheck $^{\mathrm{TM}}$. It can easily and quickly record sensory nerve conduction velocity and sensory nerve action potential of the sural nerve. It does not require specialised personnel. It has very good reproducibility and shows very good correlation with gold standard nerve conduction study. We have shown high sensitivity and specificity with very high negative predictive value against clinical examination in type 2 diabetes.

Ipswich Touch Test (ITT) is the simplest modality. The examiner touches the dorsal aspect of the 1st, 3rd, and 5th toe of the examinee bilaterally. Inability to feel at least two of these six stimuli denotes abnormal response. ITT shows good reproducibility and high correlation with the monofilament. Moreover, high sensitivity and very high specificity for DPN have been reported.

Vibratip is a tiny battery-operated handheld device testing vibration perception on the hallux and other toes. It shows excellent agreement with the neurothesiometer, the monofilament, clinical examination, and ITT. Very good accuracy for the diagnosis of DPN has also been reported.

Finally, the steel ball-bearing is a little globe testing protective sensation. Steel ball-bearings of varying diameters are applied on the plantar area over the second metatarsal head of each foot. The smallest ball-bearing felt by the examinee defines the ball-bearing score. We have reported high sensitivity and very high specificity for loss of protective sensation, as well as high sensitivity and specificity for detection of subjects with previous foot ulceration.

VG: What are the clinical implications? 
NP: New diagnostic tests should now be more widely utilised. Skin biopsy and CCM are demanding and will continue to be used in the expert setting for research purposes. They can advance our knowledge in the early stages of the natural history of DPN, in its monitoring after therapeutic interventions etc. Conversely, the indicator test Neuropad is a brilliant and well-studied screening tool with excellent reproducibility and easiness of use, lending itself also to self-examination. It has high sensitivity and negative predictive value, being very reliable in the exclusion of DPN. It may also contribute to its early diagnosis. Automated sural nerve conduction study and Ipswich Touch Test are also easy to use and valuable for DPN screening. The other tests are also useful. It now remains to be demonstrated whether all these new diagnostic tests will, indeed, increase our diagnostic rates of DPN. At any rate, they offer an important opportunity to improve DPN diagnosis and should not be ignored.

VG: Thank you so much, Nikolaos. We will be continuing with the final podcast in our Diabetic Peripheral Neuropathy collection, discussing treatment of diabetic peripheral neuropathy with Dr Uazman Alam in the next podcast.

You can listen to more podcasts by subscribing to Adis Rapid+ podcasts with your preferred podcast provider, or by visiting the website.

*In the recording of the podcast. sodium dichloride was said incorrectly, and cobalt dichloride is in fact correct. The commentary text has been changed to reflect this, however the podcast recording has not been altered.

\section{ACKNOWLEDGEMENTS}

Funding. No funding or sponsorship was received for this study or publication of this article.
Authorship. The named author meet the International Committee of Medical Journal Editors (ICMJE) criteria for authorship for this article, takes responsibility for the integrity of the work as a whole, and has given his approval for this version to be published.

Disclosures. Nikolaos Papanas has been an advisory board member of TrigoCare International, Abbott, AstraZeneca, Elpen, MSD, Novartis, Novo Nordisk, Sanofi-Aventis and Takeda; has participated in sponsored studies by Eli Lilly, MSD, Novo Nordisk, Novartis and Sanofi-Aventis; received honoraria as a speaker for AstraZeneca, Boehringer Ingelheim, Eli Lilly, Elpen, Galenica, MSD, Mylan, Novartis, Novo Nordisk, Pfizer, Sanofi-Aventis, Takeda and Vianex; and attended conferences sponsored by TrigoCare International, AstraZeneca, Boehringer Ingelheim, Eli Lilly, Novartis, Novo Nordisk, Pfizer and Sanofi-Aventis.

Compliance with Ethics Guidelines. This article does not contain any studies with human participants or animals performed by any of the authors.

Peer Review. Please note, contrary to the journal's standard single-blind peer review process, as an editorial this article underwent review by the journal's Editor-in-Chief.

Open Access. This article is distributed under the terms of the Creative Commons Attribution-NonCommercial 4.0 International License (http://creativecommons.org/licenses/ by-nc/4.0/), which permits any noncommercial use, distribution, and reproduction in any medium, provided you give appropriate credit to the original author(s) and the source, provide a link to the Creative Commons license, and indicate if changes were made. 